\title{
Microwave-Osmotic Dehydration of Cranberries under Continuous Flow Medium Spray Conditions
}

\author{
Derek Wray and Hosahalli S. Ramaswamy \\ Department of Food Science and Agricultural Chemistry, McGill University, Macdonald Campus, 21,111 Lakeshore Road, Ste. Anne \\ de Bellevue, Montreal, QC, Canada H9X 3V9 \\ Correspondence should be addressed to Hosahalli S. Ramaswamy; hosahalli.ramaswamy@mcgill.ca
}

Received 26 March 2012; Accepted 5 December 2012

Academic Editor: Tanmay Basak

Copyright (C) 2013 D. Wray and H. S. Ramaswamy. This is an open access article distributed under the Creative Commons Attribution License, which permits unrestricted use, distribution, and reproduction in any medium, provided the original work is properly cited.

\begin{abstract}
Microwave-osmotic dehydration of cranberries was evaluated under continuous flow medium spray (MWODS) conditions after some pretreatments. A central composite rotatable design was used with three input variables at five levels (temperature, $33^{\circ} \mathrm{C}-67^{\circ} \mathrm{C}$; sucrose concentration, $33^{\circ} \mathrm{B}-67^{\circ} \mathrm{B}$; and contact time, 5-55 min). Responses were moisture loss (ML), solids gain (SG), and weight reduction (WR) as well as color and texture parameters. The responses were related to process variables using response surface methodology and statistical analysis: each model was tested for lack of fit to assure nonsignificance $(P>0.05)$ and each process variable was tested for significance $(P<0.05$ or $P<0.0001)$. Temperature was found to have the most prominent effect as it was significant with all drying (ML, SG, and WR) and quality (hardness and chewiness) parameters, while contact time was found to be significant with ML and WR. Concentration wasn't found to be significant for any response. Increasing skin pretreatment severity generally promoted SG but had little effect on ML. The exception was chemical peeling, which favored ML but had no effect on SG. Overall, MWODS enables food dehydration in a much faster period of time than conventional osmotic dehydration (COD), while specifically promoting moisture loss over solids gain.
\end{abstract}

\section{Introduction}

Dehydration is one of the oldest methods of food preservation and is still prevalent in the food industry. The main objective of the dehydration process is to remove moisture to a point where the product is microbiologically and enzymatically stable and limit product deterioration during storage [1]. There is also an interest in producing dried ingredients which can then be incorporated into other products like breakfast cereals or baked goods. Drying often results in degradation of flavor volatiles, deterioration of color and texture, and an overall decrease in nutritional value, largely due to exposure to high temperatures for long periods of time in the presence of air [2]. Therefore, the key to improving the quality of dried products is to limit changes to the aforementioned quality characteristics during processing.

Osmotic dehydration (OD) represents a mild processing step in which texture is only moderately affected, nutritional value is well maintained, and the product quality, especially the color, can often be enhanced throughout the process [3]. In osmotic dehydration, the driving force is the osmotic pressure across a semipermeable membrane, which in food products is the cell wall. In addition to water exiting the plant tissue there is also a counter-current flow of solutes from the osmotic solution into the product. Furthermore, since the plant cell wall does not represent a perfect membrane, there is usually also a small leakage of low molecular weight substances such as vitamins, minerals, or organic acids which diffuse out of the cell along with the water [4]. This last diffusion tends to be insignificant in terms of mass balance but can be important in terms of quality aspects; it has been noted that anthocyanin content in cranberries after OD was $10 \%$ less than in fresh berries [5].

OD can easily be adapted to industrial applications and represents an energy efficient means to obtain an intermediate moisture food product. The energy efficiency is attributed to both the use of moderate temperatures and to the fact that there is no need to provide the latent heat of vaporization 
because water is removed by physical diffusion instead of as a vapor [6]. One drawback of conventional osmotic dehydration is the relatively slow process and therefore there has been a push to develop techniques that can be applied in conjunction with osmotic dehydration in order to speed up the process without negatively affecting the product quality [7]. The methods are applied either before or during osmotic dehydration and examples include application of vacuum $[8,9]$, ultrasound $[8]$ as well as pulsed electric field and high hydrostatic pressure [10].

In general, microwaves have been more commonly applied in conjunction with air drying in various other studies [11]. Microwave energy has proven to be effective in drying for several reasons. First, microwaves generate heat by exciting dipolar molecules (mainly water) and polarizing ionic salts; both of which try to orient themselves to the microwave field, causing rapid heating from within the sample itself, an effect known as volumetric heating [11]. During microwave-assisted dehydration there is a rapid and differential heat generated within the food product as a result of the microwave absorption, largely because the main component in the food product is water. This results in a pressure buildup within the food which in turn promotes moisture loss by forcing moisture out of the product [12]. In the case of osmotic dehydration, the increased rate of moisture loss under microwave conditions tends to reduce the inward flow of solids gain. This effect was demonstrated by Azarpazhooh and Ramaswamy, $[6,13,14]$ and Li and Ramaswamy [15-17]. In these studies, apple cylinders were treated by combined osmotic and microwave drying with a continuous flow of the osmotic medium either in an immersion (MWODI) or spray mode (MWODS). As compared to conventional osmotic dehydration, these techniques were demonstrated to provide high moisture loss, weight reduction, and ML/SG ratio along with the low solids gain and short dehydration times.

Cranberries (Vaccinium macrocarpon) represent an important cash crop in North America. The United States and Canada are the first and the second in world production and together represent about $98 \%$ of worldwide yield [5]. The vast majority of that crop is juiced, frozen, or dried prior to use, most often with sugar being incorporated because naturally cranberries are generally considered too sour and tart for direct consumption. As such, cranberries represent ideal candidates for osmotic drying because the incorporation of sucrose during the process sweetens the product as it is being dehydrated. Cranberries are also garnering interest for their health benefits which could include decreased risk of coronary heart disease [18], stroke [19], and lung cancer [20]. Moderate drying techniques are ideal in this case as high temperatures could destroy the heat labile flavonoid compounds responsible for these activities including various anthocyanins and catechins [21].

The objective of this study was to evaluate the potential for the MWODS process to be applied to a new product as each of the prior projects had been completed using apple cylinders. Further, as an extension to previous studies the focus was to quantify the effects of skin pretreatment and more specifically, MWODS process variables (temperature, contact time, sucrose concentration) on moisture loss, solids

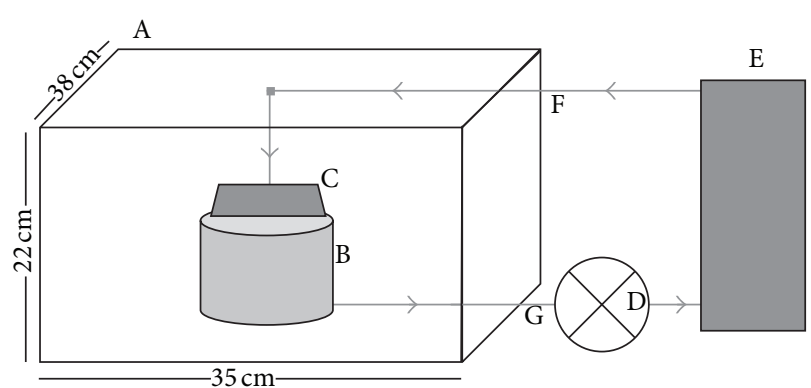

FIGURE 1: Schematic of MWODS setup: A: microwave oven cavity, B: microwave transparent sample chamber, C: spray head, D: peristaltic pump, E: water bath (containing heat exchanging coils, not pictured), and $F$ and $G$ are thermocouple measuring points immediately before and after the solution enters and leaves the microwave cavity, respectively.

gain, and weight reduction, as well as texture and color changes in cranberry samples using a central composite rotatable design (CCRD) for the experiments and response surface methodology (RSM) for analysis of results.

\section{Materials and Methods}

2.1. Raw Materials. Frozen whole cranberries (Ocean Spray Canada) were obtained from a local food service supply company and kept frozen $\left(-21\right.$ to $\left.-27^{\circ} \mathrm{C}\right)$ until use. Prior to use the cranberries were thawed for one hour at room temperature water (approximately $20^{\circ} \mathrm{C}$ ), following similar parameters to those already described for cranberries destined for OD [22]. Commercial grade sucrose (Lantic Sugar Ltd., Montreal, Qc, Canada) was used in conjunction with tap water for the osmotic solution, and the concentration was determined using a handheld refractometer (Model N2-E, ATAGO Company, Tokyo, Japan).

2.2. MWODS Setup. The experimental setup used is illustrated in Figure 1 and was the same as previously described by Azarpazhooh and Ramaswamy $[6,13]$.

\section{Experimental Procedure}

3.1. Sample Determination. In this study some initial tests were performed in order to select a sample type because previous authors [23] made reference towards difficulty in working with cranberries for an osmotic dehydration process because of the resistance against water and solids transfer provided by the waxy skin that encases the fruit. For this series of tests frozen berries were thawed in room temperature water as previously described. Pretreatments were applied when the berries were completely thawed and immediately before the samples were placed in the MWODS setup. Each of these tests was done at the same conditions $\left(50^{\circ} \mathrm{C}, 50^{\circ} \mathrm{B}\right.$, and 30 minutes), to demonstrate the effects of the skin pretreatments only. The treatments were performed as outlined here. The 7-hole treatment consisted of poking through the skin 7 times with a fine tipped needle. There were 


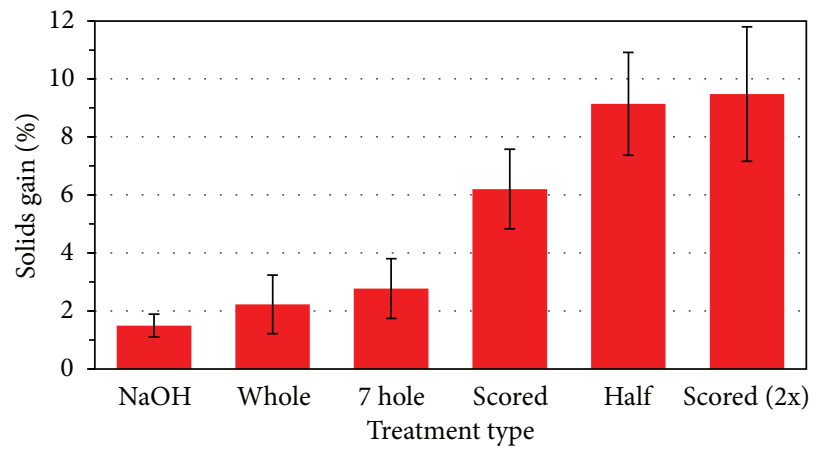

(a)

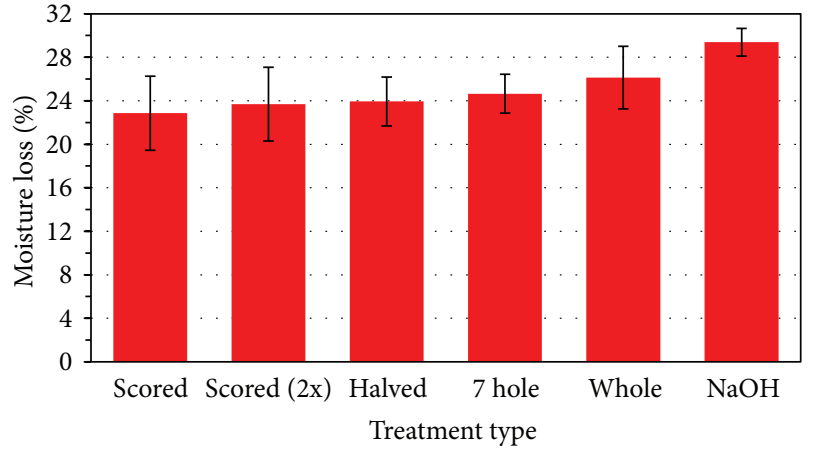

(b)

FIGURE 2: Moisture loss and solids gain for various skin pretreatments of cranberries. Average values with standard deviation shown.

5 holes places around the equator of the berry and one at each end. For scored berries the tip of a knife was moved around the long end of the berry (starting and ending at the stem) to create either a single $\mathrm{U}$-shaped cut around the fruit for the "scored" type or two intersecting U-shaped cuts for the double-scored-type pretreatment. Halved berries were cut in half along the equator by hand just prior to treatment. Finally, for chemical peeling the berries were placed in $0.5 \% \mathrm{NaOH}$ for $3 \mathrm{~min}$ at $25^{\circ} \mathrm{C}$, quickly rinsed in tap water, blotted dry, and then placed in the MWODS setup as outlined by Grabowski et al. [23]. Each sample was prepared in triplicate, and the results averaged for Figure 2.

3.2. MWODS Experiments. Individual samples of approximately $20 \mathrm{~g}$ (17-18 berries) were weighed and placed in a Nylon mesh bag to contain the sample. The system was setup and solution was preheated according to the prescribed temperature of the run type. The sample was then placed on the acrylic stage in the sample chamber in a single layer, the pump was turned on and the solution allowed to flow, and then the microwave was turned on. After the allotted time the pump was stopped and the sample was removed. The berries were then rinsed twice in a container of room temperature tap water to remove excess sugar solution from the surface of the product. The cranberries were blotted with a moist paper towel to remove surface moisture, weighed again, and were then either examined for quality parameters or dried to constant weight in an oven set at $105^{\circ} \mathrm{C}$ for approximately 24 hours [24]. The moisture content of the frozen-thawed (untreated) berries was determined to be $89.08 \%$ ( $w b)$ on average.

3.3. Experimental Design. Design Expert v6.1 (Stat-Ease Inc, Minneapolis, Minnesota, USA) was used to create a central composite rotatable design (CCRD) with three factors (temperature, sucrose concentration, and contact time) at five coded levels each $(-1.68,-1,0,1$, and 1.68). The complete experimental design with both real and coded values can be seen in Table 1.
TABLE 1: CCRD experimental design for MWODS in coded (in brackets) and real values.

\begin{tabular}{lccc}
\hline Run \# & $\begin{array}{c}\text { Temperature } \\
\left({ }^{\circ} \mathrm{C}\right)\end{array}$ & Concentration $\left({ }^{\circ} \mathrm{B}\right)$ & $\begin{array}{c}\text { Contact time } \\
(\mathrm{min})\end{array}$ \\
\hline 1 & $50(0)$ & $50(0)$ & $30(0)$ \\
2 & $50(0)$ & $67(+1.68)$ & $30(0)$ \\
3 & $50(0)$ & $50(0)$ & $30(0)$ \\
4 & $40(-1)$ & $60(+1)$ & $15(-1)$ \\
5 & $50(0)$ & $50(0)$ & $30(0)$ \\
6 & $40(-1)$ & $60(+1)$ & $45(+1)$ \\
7 & $60(+1)$ & $60(+1)$ & $15(-1)$ \\
8 & $40(-1)$ & $40(-1)$ & $15(-1)$ \\
9 & $40(-1)$ & $40(-1)$ & $45(+1)$ \\
10 & $50(0)$ & $33(-1.68)$ & $30(0)$ \\
11 & $60(+1)$ & $60(+1)$ & $45(+1)$ \\
12 & $67(+1.68)$ & $50(0)$ & $30(0)$ \\
13 & $60(+1)$ & $40(-1)$ & $15(-1)$ \\
14 & $33(-1.68)$ & $50(0)$ & $30(0)$ \\
15 & $50(0)$ & $50(0)$ & $30(0)$ \\
16 & $50(0)$ & $50(0)$ & $5(-1.68)$ \\
17 & $50(0)$ & $50(0)$ & $30(0)$ \\
18 & $60(+1)$ & $40(-1)$ & $45(+1)$ \\
19 & $50(0)$ & $50(0)$ & $30(0)$ \\
20 & $50(0)$ & $50(0)$ & $55(+1.68)$ \\
\hline
\end{tabular}

\section{Quality Analysis}

For each run type, partial samples were taken for quality analysis, while the rest of the samples were dried until completion for the determination of solids gain and moisture loss.

4.1. Color Measurement. Color values of the MWODStreated samples were determined in the $L^{*}, a^{*}, b^{*}$ system using a tristimulus Minolta Chroma Meter (Minolta Corp., Ramsey, NJ, USA). The Chroma Meter was warmed up 20 
TABLE 2: CCRD run numbers with results for post-MWODS drying and quality parameters (average values with standard deviation shown).

\begin{tabular}{|c|c|c|c|c|c|}
\hline Run \# & ML (\%) & SG (\%) & WR (\%) & Hardness $(\mathrm{N})$ & Chewiness (mJ) \\
\hline 1 & $22.2(3.10)$ & $2.73(0.53)$ & $22.4(2.68)$ & $162(41.4)$ & $89.9(41.3)$ \\
\hline 2 & $21.7(1.16)$ & $2.74(0.16)$ & $21.9(1.10)$ & $92.2(25.8)$ & $24.4(14.5)$ \\
\hline 3 & $23.8(0.60)$ & $2.75(0.51)$ & $24.0(0.31)$ & $154(14.8)$ & $64.8(18.4)$ \\
\hline 4 & $14.3(1.29)$ & $1.92(0.22)$ & $15.3(1.30)$ & $206(61.5)$ & $79.4(28.4)$ \\
\hline 5 & $22.3(2.35)$ & $2.28(0.27)$ & $22.9(2.32)$ & $157(22.2)$ & $62.6(33.1)$ \\
\hline 6 & $17.6(1.77)$ & $2.31(0.21)$ & $18.2(1.94)$ & 205 (27.9) & $94.2(22.5)$ \\
\hline 7 & $22.5(2.20)$ & $1.99(0.66)$ & $23.5(1.43)$ & $162(24.9)$ & $66.6(14.0)$ \\
\hline 8 & $19.8(1.26)$ & $2.65(0.86)$ & $14.3(2.75)$ & $155(24.5)$ & $67.4(13.9)$ \\
\hline 9 & $22.0(3.55)$ & $1.79(0.32)$ & $23.2(3.71)$ & $257(33.6)$ & $184(31.0)$ \\
\hline 10 & $19.8(1.26)$ & $2.75(0.31)$ & $20.0(1.56)$ & $178(38.0)$ & $73.0(30.0)$ \\
\hline 11 & $29.7(1.89)$ & $3.27(1.03)$ & $29.4(2.83)$ & $125(14.1)$ & $36.0(6.26)$ \\
\hline 12 & $21.3(3.09)$ & $5.91(1.30)$ & $18.4(2.86)$ & $156(66.8)$ & $66.5(48.0)$ \\
\hline 13 & $23.7(1.18)$ & $2.20(0.13)$ & $24.5(1.05)$ & $180(39.4)$ & $66.3(32.1)$ \\
\hline 14 & $17.0(3.65)$ & $2.36(2.34)$ & $17.6(3.62)$ & $239(36.4)$ & $143(23.6)$ \\
\hline 15 & $22.1(2.37)$ & $2.34(0.98)$ & $22.6(3.32)$ & $156(27.1)$ & $49.9(19.3)$ \\
\hline 16 & $17.3(2.11)$ & $2.10(0.58)$ & $18.3(2.50)$ & $208(20.4)$ & $76.5(12.5)$ \\
\hline 17 & $21.7(1.08)$ & $2.49(0.40)$ & $22.1(0.72)$ & $183(24.8)$ & $105(28.0)$ \\
\hline 18 & $30.8(2.87)$ & $5.41(2.62)$ & $28.3(1.49)$ & $108(17.6)$ & $34.8(10.6)$ \\
\hline 19 & $25.1(2.07)$ & $1.93(0.42)$ & $26.1(1.68)$ & $194(33.3)$ & $77.0(32.0)$ \\
\hline 20 & $24.0(1.14)$ & $2.34(0.52)$ & $24.6(0.78)$ & $154(17.2)$ & $78.9(35.2)$ \\
\hline Fresh & & & & $293(71.9)$ & $121(69.7)$ \\
\hline
\end{tabular}

minutes prior to use and calibrated against a white standard. Eight measurements were taken with each sample, and the values were averaged in order to obtain the $L^{*}$ (lightness), $a^{*}$ (green $(-)$ to red $(+)$ ), and $b^{*}$ (blue $(-)$ to yellow $(+)$ ) values of the individual trials. The $\Delta E$ (total color change), $C^{*}$ (chroma), and $H^{\circ}$ (hue angle) were also determined according to the following equations [25]:

$$
\begin{gathered}
\Delta E=\sqrt{\left(L_{0}-L\right)^{2}+\left(a_{0}-a\right)^{2}+\left(b_{0}-b\right)^{2}}, \\
\text { Chroma }\left(C^{*}\right)=\sqrt{a^{2}+b^{2}}, \\
\text { Hue Angle }\left(H^{\circ}\right)=\tan ^{-1} \frac{b}{a}
\end{gathered}
$$

where $L_{0}, a_{0}$, and $b_{0}$ and $L, a$, and $b$ represent the values of the fresh and processed samples, respectively.

4.2. Texture Analysis. Texture profile analysis (TPA) of both the MWODS-treated samples and fresh berries was performed using a TA.XTPlus Texture Analyser (Stable Microsystems, Surrey, UK). TPA allows for the determination of a wide range of responses such as adhesiveness, chewiness, cohesiveness, fracturability, gumminess, hardness, and springiness [26]. In this study hardness and chewiness were selected as parameters of interest as they represent quality indicators one would experience during mastication of a raisin-type semidried product. Hardness was taken as the peak of the first curve $(\mathrm{N})$, while chewiness $(\mathrm{mJ})$ is calculated as the gumminess multiplied by springiness, as defined by Bourne [26]. The analysis was performed with eight replicates, and the average values (with standard deviation) are presented in Table 2. Analysis was performed by means of TPA using a flat bottom probe $(25 \mathrm{~mm}$ diameter $)$ with pretest speed of $1 \mathrm{~mm} / \mathrm{sec}$, test speed of $5 \mathrm{~mm} / \mathrm{sec}$ and posttest speed of $5 \mathrm{~mm} / \mathrm{sec}$. The target was a distance of $10 \mathrm{~mm}$, which represents the $50 \%$ of the average diameter of the cranberries tested. These settings were used with guidance from Rahman and Al-Farsi [27], who used TPA on a similar dried product (dates).

\section{Osmotic Dehydration Responses and Data Analysis}

To analyze the mass exchange that took place during osmotic dehydration, the parameters of moisture loss (ML), solids gain (SG), and weight reduction (WR) were calculated according to the following equations:

$$
\begin{gathered}
\% \mathrm{ML}=100 \frac{M_{0} x_{0}-M_{t} x_{t}}{M_{0}}, \\
\% \mathrm{SG}=100 \frac{M_{0} \mathrm{~s}_{0}-M_{t} \mathrm{~s}_{t}}{M_{0}}, \\
\% \mathrm{WR}=100 \frac{M_{0}-M_{t}}{M_{0}},
\end{gathered}
$$

where $M_{0}$ and $M_{t}$ are the total sample mass as time 0 and time $t$, respectively; $x_{0}$ and $x_{t}$ are the moisture fractions (kg/kg wet basis) at time 0 and time $t$, respectively; $s_{0}$ and $s_{t}$ are the solid fractions $(\mathrm{kg} / \mathrm{kg}$ wet basis) at time 0 and time $t$, respectively. These equations assume a one-way mass 
transfer of sucrose into the product (i.e., there is no significant leeching of solids from the sample into the solution). These parameters were collected by weighing the samples before MWODS treatment $\left(M_{0}\right)$, after treatment $\left(M_{t}\right)$. The initial solids fraction $\left(s_{0}\right)$ was calculated by difference from the moisture content of fresh cranberries, and the posttreatment solids content $\left(s_{t}\right)$ was defined as the mass gained after drying to constant weight. Each run was completed in triplicate with average values used for data analysis.

\section{Results and Discussion}

6.1. Pretreatment. For most of the treatment types the moisture loss for the various skin treatments remained near constant and certainly within the error of the samples (Figure $2)$. The only exception is the chemically $(\mathrm{NaOH})$ peeled sample, which did have a slightly higher moisture loss when compared to the rest. Interestingly, the halved berries provided nearly the same amount of moisture loss when compared to the whole berry. This is in contrast to Grabowski et al. [23], who found that cutting the berries in half increased the moisture diffusion by about 100-fold when compared to the mass transfer through the cranberry skin and overall by about a $4.5 \%$ increase in moisture loss over the length of the process. There are a few possible explanations for this change, including a smaller solution-sample contact area in a spray setup when compared to a traditional immersion setup. This is particularly important when you consider the positioning of the cranberry halves under the spray head. While each sample was placed facing "up" to sit like a bowl on the sample stage, if a stream of spray did not exactly enter and fill that piece, it would not have nearly the same, effect as if the sample was completely submerged in the solution.

The solids gain for the most part increased with the level of destruction to the skin. The lone exception is that halved berries accounted for less solids gain, on average, than the double scored samples, although the variation is within the error. Also of interest is the fact that the chemically peeled samples showed no increased absorption of sucrose when compared to untreated samples. This suggests that while removing the waxy layer allows water to more easily traverse the skin, the skin tissue that remains may still be a physical barrier that prevents solids uptake.

Since any of the previous pretreatments were considered to be fully satisfactory, it was decided to use the untreated whole berries for two key reasons. First, the unaltered skin on the whole sample provided an interesting property in that it would limit the uptake of solute. This is of particular interest in osmotic dehydration where it is optimal to provide a high $\mathrm{ML} / \mathrm{SG}$ ratio, that is, providing the highest possible moisture loss while limiting solids gain [6]. Secondly, while the $\mathrm{NaOH}$ treatment increased the moisture loss and potential ML/SG over the untreated berries, it was concluded that the effect was not enough to justify the use of synthetic chemicals in the process. These results agree with another conclusion from a similar study where it was determined that chemical pretreatment provided no significant influence on water transfer during osmotic dehydration of cranberries [28].
However, throughout the study it was observed that there were random breaks in the skin likely due to the freezethaw process. This possibly contributed to the variation of the results and would have therefore reduced the accuracy of the predicting model. In future studies it may be advantageous to use a skin pretreatment in order to minimize these effects.

\section{Response Surface Methodology (MWODS)}

The experimental design for this set of experiments differed from traditional studies of osmotic dehydration. Because conventional osmotic dehydration is a relatively slow process, studies tend to fix variables such as temperature, concentration, and flow rate and measure samples at a given interval until a targeted equilibrium is reached [6]. Because of the requirement to test the statistical significance of each of the process variables (temperature, concentration and contact time) in a manageable number of experiments, a central composite rotatable design (CCRD) was employed and provided a set of 20 individual run types to perform that independently tests the effect of that parameter on the response (Table 1). By design, the program does not require duplicates of each individual run, as there are six repeated run types (center points) which determine the variability of the process and the lack of fit of the model. However, due to the intrinsic variability of microwaves in drying, each run type was performed in triplicate and the mean value entered into Design Expert for analysis (Table 2).

In working with a CCRD design one of the most important steps is to determine the statistical significance of the model and each of the parameters uses analysis of variance (ANOVA). The models selected for each of the responses in this study were selected according to their statistical significance and lack of fit values (Table 3). For moisture loss and weight reduction, this represented the linear model (both $P<0.0001$ ), while solids gain and hardness used a quadratic model (both $P<0.05$ ), and finally chewiness was best represented by an interaction $(2 \mathrm{FI})$ model $(P<$ $0.05)$. For each of the models it is critical to ensure that the lack of fit is not significant $(P>0.05)$; this value for each model is shown along with correlation coefficients in Table 3. Lack of fit is determined by testing the repeated center points, and an insignificant lack of fit tells us that the model is able to adequately predict the response variables. Using the predicting equations determined by Design Expert (Table 3), we can predict the outcome for responses such as moisture loss, solids gain, weight reduction, hardness, or chewiness depending on a function of input process variables.

\section{Effects of Individual Process Variables}

8.1. Moisture Loss. Beginning with moisture loss, it was found that only the linear terms of temperature $(P<0.0001)$ and contact time $(P<0.05)$ had a significant effect on ML. No interactions or quadratic terms were found to have any significant effect. Based on the sum of squares produced by ANOVA (not shown), it can be stated that temperature had a larger effect than contact time in terms of determining 


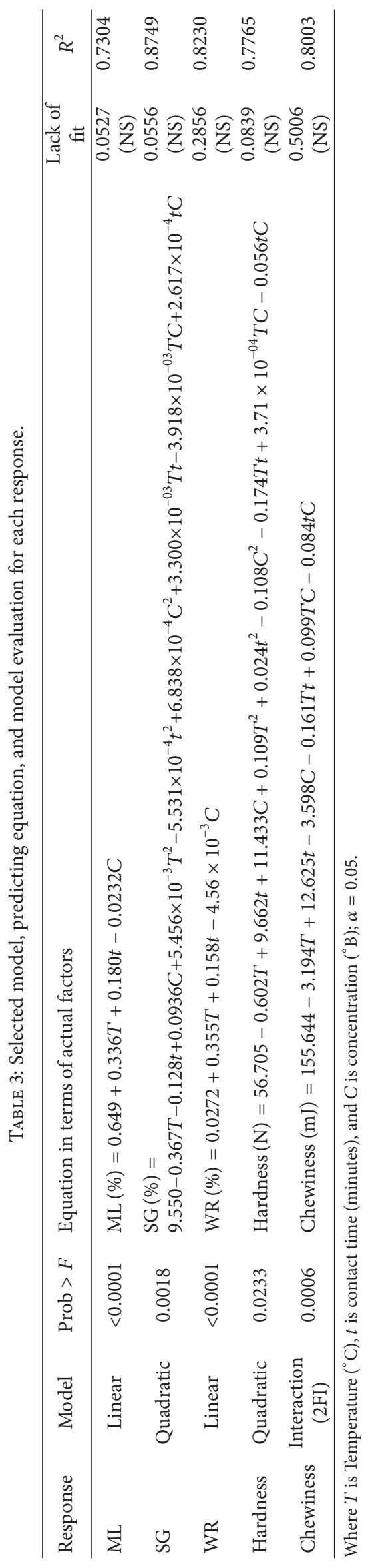


moisture loss during MWODS. Since the coefficients for both of these terms were positive, it can be further stated that an increase in either of the variables would result in higher moisture loss. This makes sense in the context of previous studies as osmotic dehydration has been previously described as a temperature-dependant process [15]. Response surface plots showing the effect on moisture loss of increasing temperature (Figure 3(a)) and concentration (Figure 3(d)) as a function of a time show that while increasing temperature has a large effect on the ML whole cranberries over the length of the process, concentration has little to no effect. In this case, moisture loss remains relatively stable as concentration increases and changes mainly with longer contact times. Many studies have formed the general conclusion that increasing temperature or contact time increased moisture loss whether it is in an immersion or spray setup and with or without microwaves being applied [1, 6, 13, 15-17]. The effects of temperature on moisture loss can be largely attributed to three key points. First, higher temperatures reduce the solution viscosity, allowing better contact between the sample and the osmotic solution, higher mobility of water molecules, and overall higher extraction ability of the solution. This effect is particularly important at high sucrose concentrations where viscosity can cause issues in a spraybased system [6]. Second, higher temperatures cause the individual cells in the sample to swell, thereby increasing the moisture permeability across the cell wall, which normally moderates moisture movement. Finally, thermal energy promotes moisture diffusion within the sample itself, which means that the water molecules can more easily reach the sample surface and be removed into the osmotic solution. These three causes were determined by several studies $[6,15]$. While contact time was found to provide a significant effect on the moisture loss of the sample, it was lower than that of temperature. This is in contrast to some previous studies on both conventional [1, 15] and microwave-assisted [6] osmotic dehydration; all of which concluded that contact time had the largest influence on moisture loss. Similarly, past studies concluded that concentration does provide a significant impact on moisture loss, just not as large as that of temperature [29]. These incongruities with past results were perhaps caused by the effect of the cranberry skin, which is naturally intended to keep water inside the fruit as well as foreign substances out. If on the skin, which is at the junction of the fruit flesh and the osmotic solution, there was a layer of sucrose that formed near the beginning of the run; it is possible that this would provide enough resistance to moisture removal that longer run times or increased concentration had no appreciable effect on the overall moisture loss.

8.2. Solids Gain. In terms of solids (sucrose) gain by the cranberry samples only the temperature had a significant effect, where it was found to be significant $(P<0.05)$ at both the linear and the quadratic levels. The interaction effect between temperature and contact time was also found to be significant $(P<0.05)$. Like moisture loss, all of the significant terms for solids gain had positive coefficients, signifying that increasing temperature or contact time would results in higher solids gain. This effect can be visualized in Figure 3(b), where the interaction effect between contact time and temperature shows an increase in either of these variables resulting in increasing solids gain. These results agree with the general conclusions of several studies where increased temperature also increases the solids gain of the product $[1,6,15,30]$. The concentration of sucrose in the osmotic solution was found to be insignificant and actually slightly decreased the solids gain. This trend can be seen in Figure 3(e), where higher concentrations actually result in lower solids gain throughout the range, an effect that partially disagrees with the majority of published reports. It has been seen in past studies that solids gain increases with solute concentration until extremely high concentrations at which point solids gain is often lower than at more moderate concentrations. This effect is likely caused by the formation of a dense superficial layer of solute at the surface of the sample, which then blocks further uptake of the solute [15]. While normally only evident at higher concentrations in the case of whole cranberries it is likely that the skin provides an additional barrier which blocks solids uptake in the fruit and facilitates the formation of the dense surface layer of solute, thereby slowing the uptake of solids over a broader range of concentrations instead of only at high concentrations.

8.3. Weight Reduction. Since weight reduction is determined by the moisture loss mediated by the solids gain and the moisture loss is typically larger than the solids gain by an order of magnitude, it is expected that the responses of weight reduction and moisture loss will follow a similar trend [6]. Therefore, it is not surprising to see that for weight reduction, like moisture loss, only the linear terms of temperature $(P<0.0001)$ and contact time $(P<0.05)$ were significant, while all of the quadratic and interaction effects proved to be insignificant. For the linear terms, the positive coefficients confirm that increasing either the temperature or contact time would increase the level of weight reduction, much like moisture loss. In general, increasing temperature will increase the weight reduction over the length of the MWODS process (Figure 3(c)), which shows that higher temperature tends to favour moisture loss over solids gain, thereby promoting moisture loss. It can also be concluded that concentration had little to no effect on weight reduction (Figure 3(f)), where the increase was due to increased contact time alone. This is nearly identical to response of the same interaction for moisture loss (Figure 3(d)).

8.4. Color. In this study, none of the parameters (or models) had any significant effect on the change in color measurements of the MWODS samples (in terms of $L^{*} a^{*} b^{*}$, Chroma $\left(C^{*}\right)$, Hue Angle $\left(H^{\circ}\right)$, or total color change $(\Delta E)$ values). As such, none of these values are presented here. For these responses, statistical analysis dictated that the mean change in the value better represented the predicted outcome than any linear (or higher) model. There are two possible conclusions from this outcome; first there is the possibility that there was no significant change in the color throughout the MWODS 


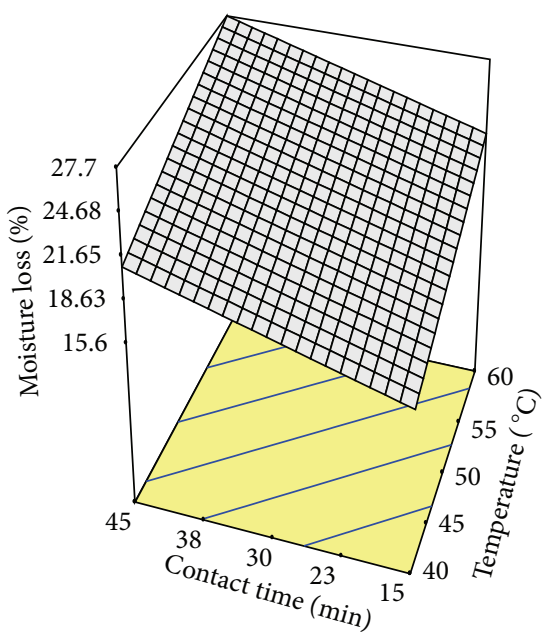

(a)

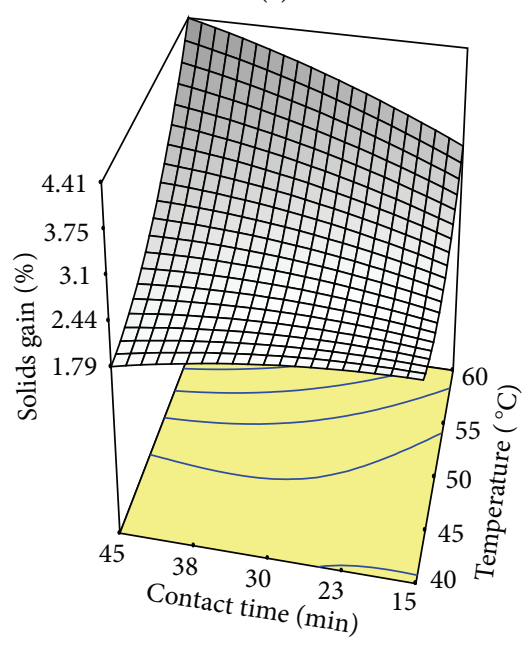

(b)

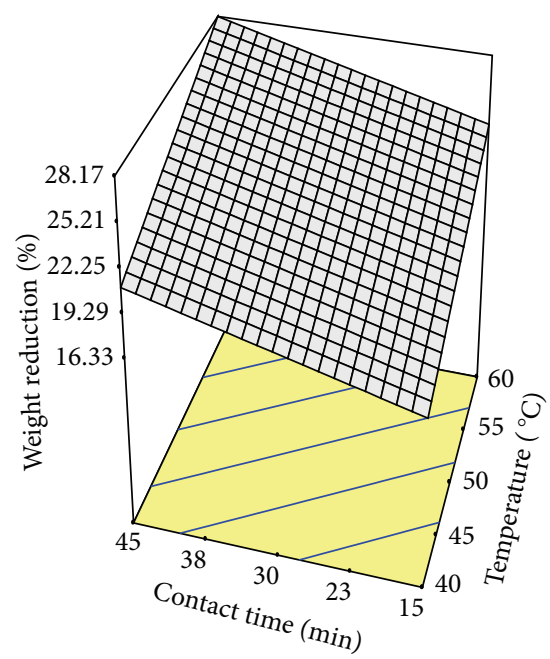

(c)

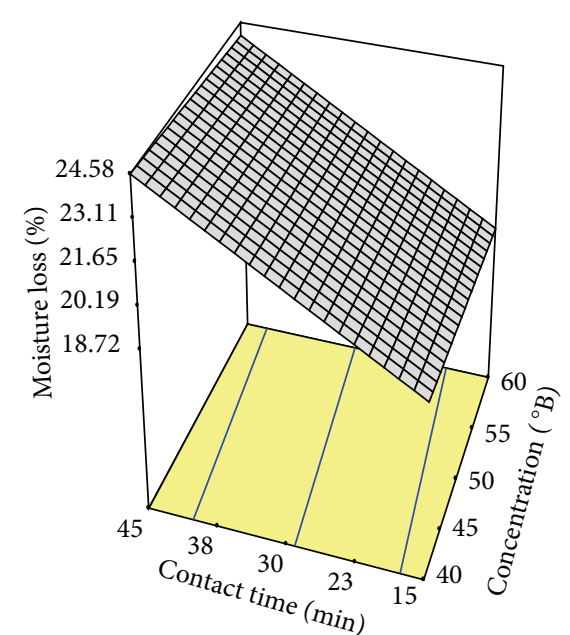

(d)

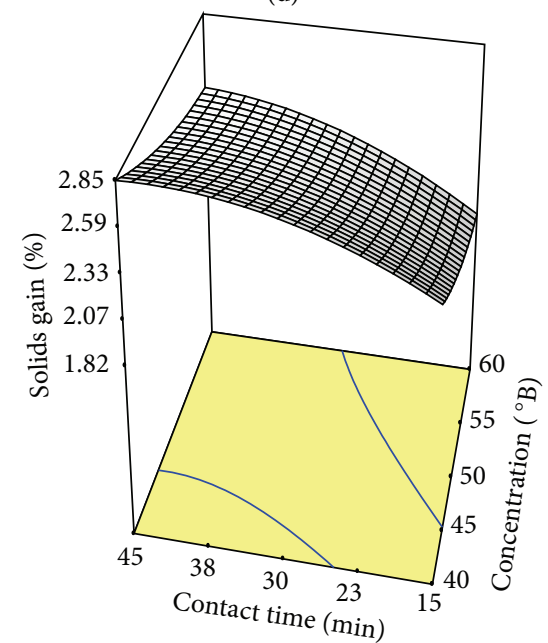

(e)

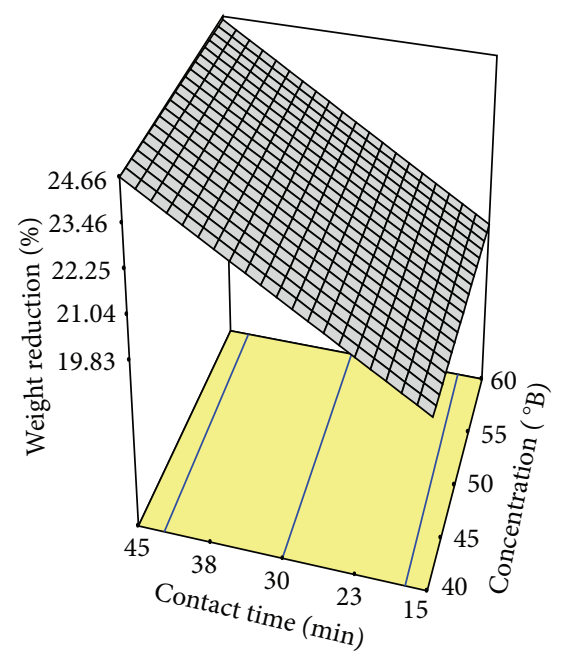

(f)

FIgURE 3: Response surface plots for dehydration parameters (ML, SG, and WR). For variable temperature plots (a-c), concentration was kept constant at its center point $\left(50^{\circ} \mathrm{B}\right)$, while for variable concentration plots $(\mathrm{d}-\mathrm{f})$, temperature was kept at its center point $\left(50^{\circ} \mathrm{C}\right)$. 


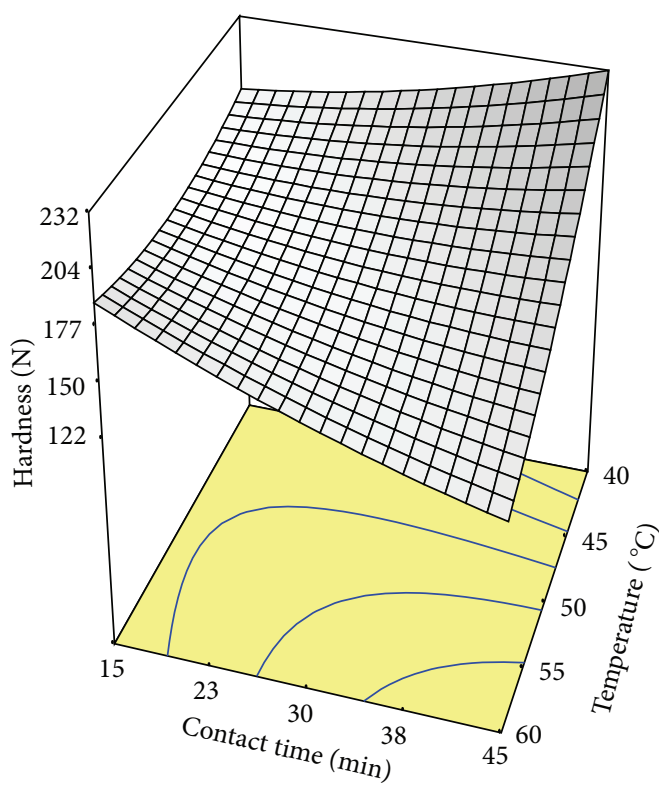

(a)

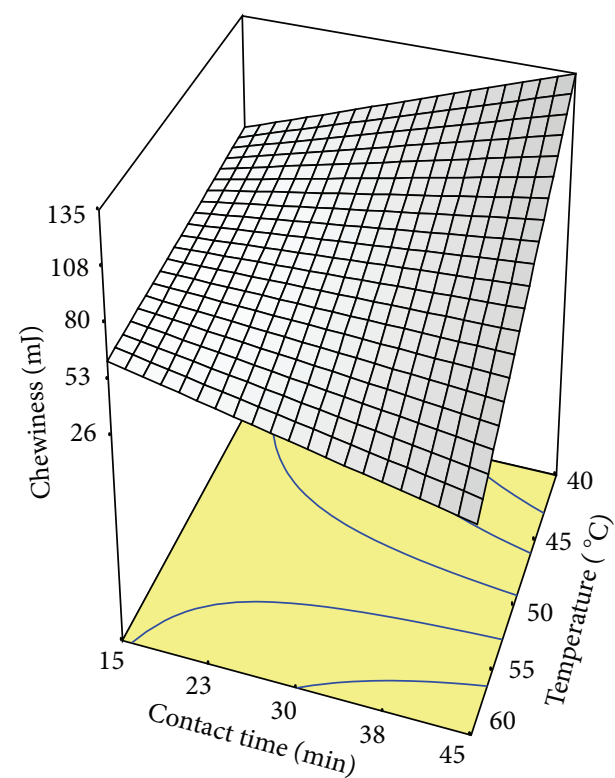

(b)

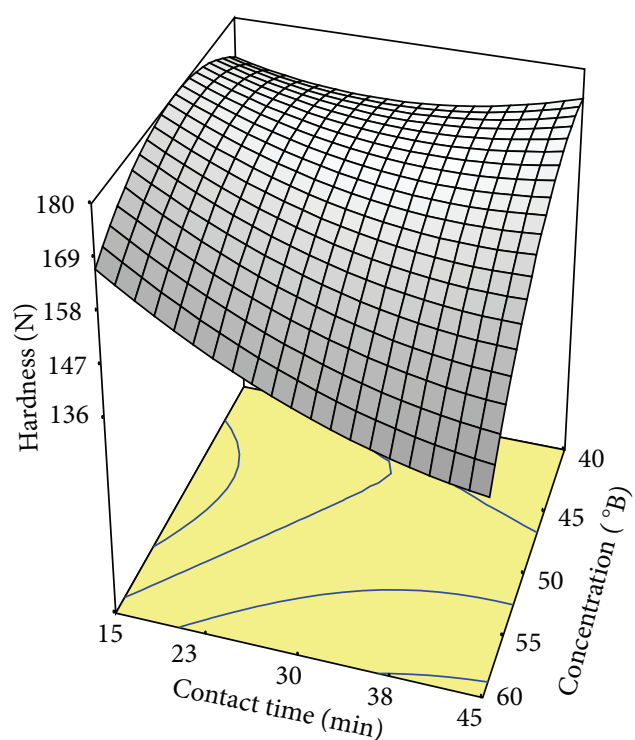

(c)

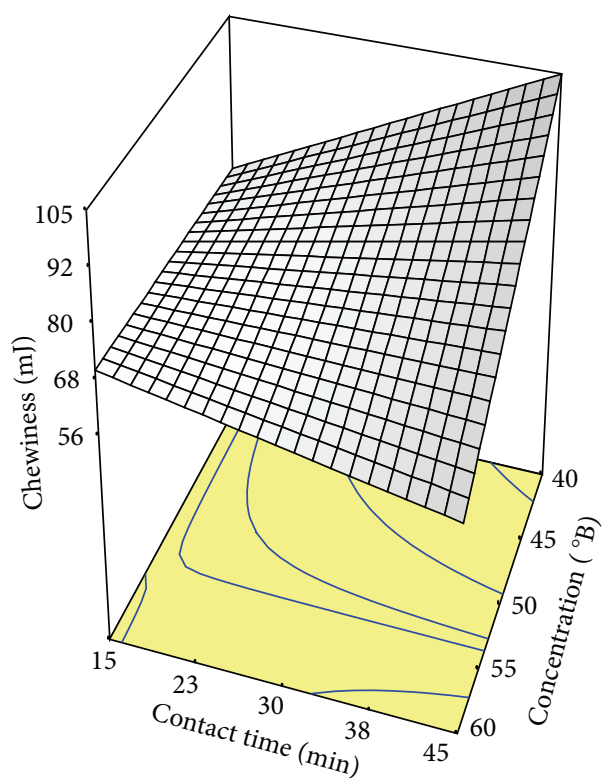

(d)

FIGURE 4: Response surface plots for quality parameters (Hardness and Chewiness). For variable temperature plots (a, b), concentration was kept constant at its center point $\left(50^{\circ} \mathrm{B}\right)$, while for variable concentration plots $(\mathrm{c}, \mathrm{d})$, temperature was kept at its center point $\left(50^{\circ} \mathrm{C}\right)$.

process. This is quite possible as typically these quality tests have only been performed on finished products (after secondary drying). The second possible conclusion is that even the least severe MWODS treatment made some change to the color properties, and this change did not increase with increased treatment severity.

8.5. Texture. Like color measurements, mechanical properties are typically only tested after the finished drying step, but the interest here was to see if the MWODS process caused any significant change in the textural attributes as a standalone process. For both chewiness and hardness the linear effect of temperature provided the only significant process variable effect, where $P<0.0001$ and $P<0.05$ for hardness and chewiness, respectively. For both of these responses the interaction effect between temperature and contact time proved to be significant on a $P<0.05$ level; these interactions can be seen in Figures 4(a) and 4(b). The variable coefficients for these effects are negative, indicating that increasing either temperature or contact time actually decreases the hardness and chewiness when compared to the fresh product. This agrees with previous studies that have concluded that osmotically dried samples become softer and more plastic as 
samples losing these characteristics will become softer and less elastic, respectively [31]. It has been described that the mechanical properties of dried products are closely related to the physical state and structure developed as a result of typical deformations such as cell shrinking or swelling, changes in intercellular spaces (volume), and rupturing of cell bonds [32]. These changes influence mechanical behaviour in several fashions; first, air and liquid volumes within the sample change as solute is taken into the food, and the size of the product can be altered as well [9]. Secondly, detachment of the middle lamella of plant cells results in the loss of cell turgor which in turn affects the cell wall and puncture strength [33]. In examining the relationship between cell structure and texture, it would be a logical conclusion that higher temperatures and concentrations would exacerbate cell damage and therefore further affect the mechanical properties, which concur with the trends seen here. The effects of concentration of textural responses were more mixed.

\section{Conclusions}

Overall the level of destruction of cranberry skin increased moisture and solids diffusion, however, not to a significant extent. Therefore, the decision to leave the cranberries as a whole with no skin pretreatment was done in part to omit chemical peeling techniques and ease of processing but also to observe the ability of the MWODS system to overcome an the effect of a natural moisture barrier that is prevalent in small berries such as cranberries. A CCRD model combined with RSM analysis was used to determine the effect of individual process parameters (temperature, sucrose concentration, and contact time) on a series of responses (ML, SG, WR, hardness, and chewiness). The CCRD model was used to reduce the number of experiments required while still testing each variable independently. Overall, it was found that temperature was a significant factor for every response, where higher temperatures produced higher moisture loss, solids gain, and weight reduction along with reduced hardness and chewiness. Additionally, contact time was found to be a significant positive independent variable for ML, SG, and WR; implying increasing process time would in turn enhance these responses, while longer process times tended to decrease textural characteristics. Concentration effects tended to be minimal for each of the responses, which can be largely attributed to the unique properties of the cranberry skin. No model was found to be significant for any color change response, and therefore the color destruction during MWODS was insignificant. Overall it was found that the MWODS process is suitable for products like small berries; however, removal of the skin would make it easier to further model the process.

\section{References}

[1] N. H. Van Nieuwenhuijzen, M. R. Zareifard, and H. S. Ramaswamy, "Osmotic drying kinetics of cylindrical apple slices of different sizes," Drying Technology, vol. 19, no. 3-4, pp. 525-545, 2001.
[2] I. Alibas, "Microwave, air and combined microwave-air-drying parameters of pumpkin slices," LWT_Food Science and Technology, vol. 40, no. 8, pp. 1445-1451, 2007.

[3] C. Lerici, G. Pinnavaia, M. Rosa, and L. Bortolucci, "Osmotic dehydration of fruit influence of osmotic agents on drying behaviour and product quality," Journal of Food Science, vol. 50, no. 5, pp. 1217-1219, 1985.

[4] D. Torreggiani, "Osmotic dehydration in fruit and vegetable processing," Food Research International, vol. 26, no. 1, pp. 59-68, 1993.

[5] S. Grabowski, M. Marcotte, M. Poirier, and T. Kudra, "Drying characteristics of osmotically pretreated cranberries-energy and quality aspects," Drying Technology, vol. 20, no. 10, pp. 1989-2004, 2002.

[6] E. Azarpazhooh and H. S. Ramaswamy, "Evaluation of factors influencing microwave osmotic dehydration of apples under continuous flow medium spray (MWODS) conditions," Food and Bioprocess Technology, vol. 5, no. 4, pp. 1265-1277, 2010.

[7] N. K. Rastogi, K. S. M. S. Raghavarao, K. Niranjan, and D. Knorr, "Recent developments in osmotic dehydration: methods to enhance mass transfer," Trends in Food Science and Technology, vol. 13, no. 2, pp. 48-59, 2002.

[8] Y. Deng and Y. Zhao, "Effects of pulsed-vacuum and ultrasound on the osmodehydration kinetics and microstructure of apples (Fuji)," Journal of Food Engineering, vol. 85, no. 1, pp. 84-93, 2008.

[9] P. Fito, "Modelling of vacuum osmotic dehydration of food," Journal of Food Engineering, vol. 22, no. 1-4, pp. 313-328, 1994.

[10] K. A. Taiwo, A. Angersbach, B. I. O. Ade-Omowaye, and D. Knorr, "Effects of pretreatments on the diffusion kinetics and some quality parameters of osmotically dehydrated apple slices," Journal of Agricultural and Food Chemistry, vol. 49, no. 6, pp. 2804-2811, 2001.

[11] V. Orsat, W. Yang, V. Changrue, and G. S. V. Raghavan, "Microwave-assisted drying of biomaterials," Food and Bioproducts Processing, vol. 85, no. 3, pp. 255-263, 2007.

[12] M. E. Sosa-Morales, L. Valerio-Junco, A. López-Malo, and H. S. García, "Dielectric properties of foods: reported data in the 21st century and their potential applications," LWT-Food Science and Technology, vol. 43, no. 8, pp. 1169-1179, 2010.

[13] E. Azarpazhooh and H. S. Ramaswamy, "Microwave-osmotic dehydration of apples under continuous flow medium spray conditions: comparison with other methods," Drying Technology, vol. 28, no. 1, pp. 49-56, 2010.

[14] E. Azarpazhooh and H. S. Ramaswamy, "Evaluation of diffusion and Azuara models for mass transfer kinetics during microwave-osmotic dehydration of apples under continuous flow medium-spray conditions," Drying Technology, vol. 28, no. 1, pp. 57-67, 2010.

[15] H. Li and H. Ramaswamy, "Osmotic dehydration of apple cylinders: I. Conventional batch processing conditions," Drying Technology, vol. 24, no. 5, pp. 619-630, 2006.

[16] H. Li and H. Ramaswamy, "Osmotic dehydration of apple cylinders: II. Continuous medium flow heating conditions," Drying Technology, vol. 24, no. 5, pp. 631-642, 2006.

[17] H. Li and H. S. Ramaswamy, "Osmotic dehydration of apple cylinders: III. Continuous medium flow microwave heating conditions," Drying Technology, vol. 24, no. 5, pp. 643-651, 2006.

[18] P. Knekt, R. Järvinen, A. Reunanen, and J. Maatela, "Flavonoid intake and coronary mortality in Finland: a cohort study," British Medical Journal, vol. 312, no. 7029, pp. 478-481, 1996. 
[19] S. O. Keli, M. G. L. Hertog, E. J. M. Feskens, and D. Kromhout, "Dietary flavonoids, antioxidant vitamins, and incidence of stroke: the Zutphen study," Archives of Internal Medicine, vol. 156, no. 6, pp. 637-642, 1996.

[20] P. Knekt, R. Järvinen, R. Seppänen et al., "Dietary flavonoids and the risk of lung cancer and other malignant neoplasms," American Journal of Epidemiology, vol. 146, no. 3, pp. 223-230, 1997.

[21] W. Zheng and S. Y. Wang, "Oxygen radical absorbing capacity of phenolics in blueberries, cranberries, chokeberries, and lingonberries," Journal of Agricultural and Food Chemistry, vol. 51, no. 2, pp. 502-509, 2003.

[22] J. Yongsawatdigul and S. Gunasekaran, "Microwave-vacuum drying of cranberries: part I. Energy use and efficiency," Journal of Food Processing and Preservation, vol. 20, no. 2, pp. 121-143, 1996.

[23] S. Grabowski, M. Marcotte, D. Quan et al., "Kinetics and quality aspects of Canadian blueberries and cranberries dried by osmoconnective method," Drying Technology, vol. 25, no. 2, pp. 367-374, 2007.

[24] AOAC, "Moisture in dried fruits," in Official Methods of the Association of Official Analytical Chemists, AOAC International, Maryland, Md, USA, 17th edition.

[25] N. Maftoonazad and H. S. Ramaswamy, "Effect of pectin-based coating on the kinetics of quality change associated with stored avocados," Journal of Food Processing and Preservation, vol. 32, no. 4, pp. 621-643, 2008.

[26] M. Bourne, "Principles of objective texture measurement," in Concept and Measurement, pp. 114-117, Academic Press, San Diego, Calif, USA, 1982.

[27] M. S. Rahman and S. A. Al-Farsi, "Instrumental texture profile analysis (TPA) of date flesh as a function of moisture content," Journal of Food Engineering, vol. 66, no. 4, pp. 505-511, 2005.

[28] P. S. Sunjka, T. J. Rennie, C. Beaudry, and G. S. V. Raghavan, "Microwave-convective and microwave-vacuum drying of cranberries: a comparative study," Drying Technology, vol. 22, no. 5, pp. 1217-1231, 2004.

[29] F. Nsonzi and H. S. Ramaswamy, "Osmotic dehydration kinetics of blueberries," Drying Technology, vol. 16, no. 3-5, pp. 725-741, 1998.

[30] H. N. Lazarides, E. Katsanidis, and A. Nickolaidis, "Mass transfer kinetics during osmotic preconcentration aiming at minimal solid uptake," Journal of Food Engineering, vol. 25, no. 2, pp. 151-166, 1995.

[31] A. Monsalve-Gonzalez, V. Gustavo, and P. Ralph, "Mass transfer and textural changes during processing of apples by combined methods," Journal of Food Science, vol. 58, no. 5, pp. 1118-1124, 1993.

[32] C. Contreras, M. E. Martín-Esparza, N. Martínez-Navarrete, and A. Chiralt, "Influence of osmotic pre-treatment and microwave application on properties of air dried strawberry related to structural changes," European Food Research and Technology, vol. 224, no. 4, pp. 499-504, 2007.

[33] A. Chiralt and P. Talens, "Physical and chemical changes induced by osmotic dehydration in plant tissues," Journal of Food Engineering, vol. 67, no. 1-2, pp. 167-177, 2005. 

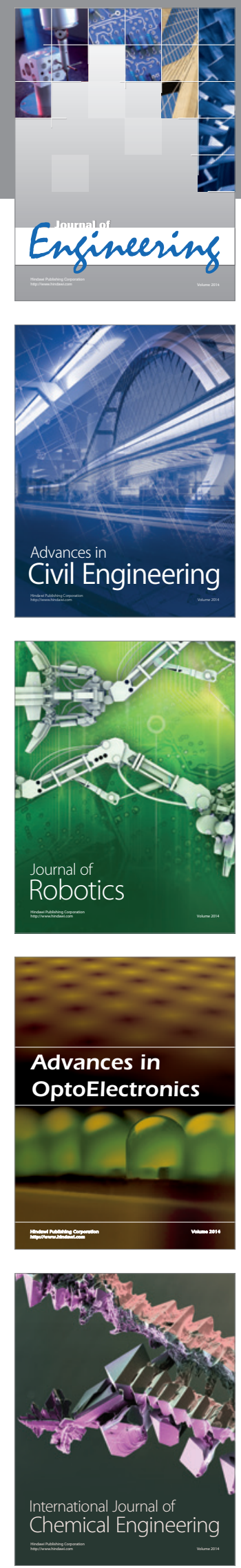

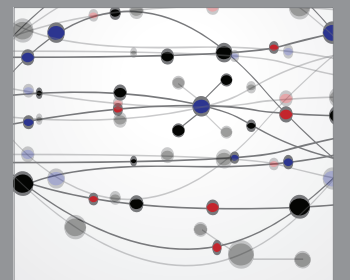

The Scientific World Journal
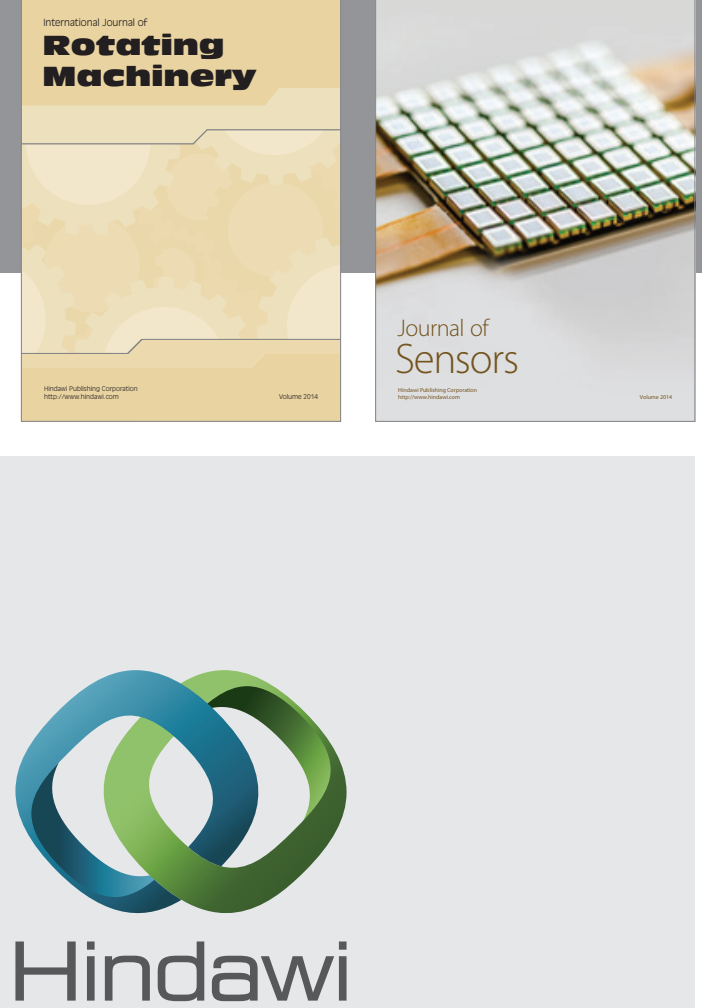

Submit your manuscripts at http://www.hindawi.com
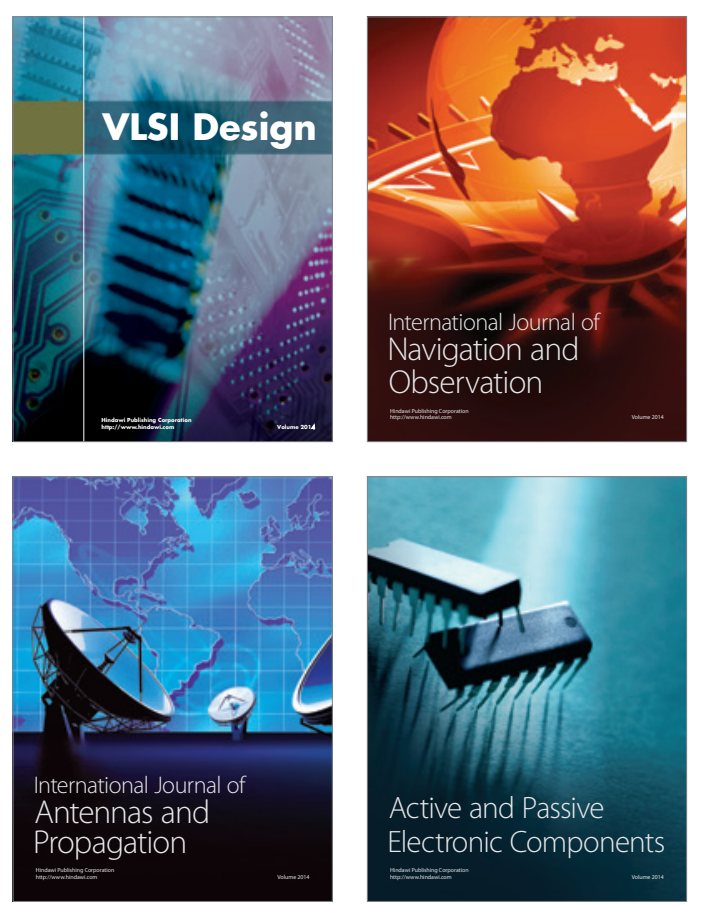
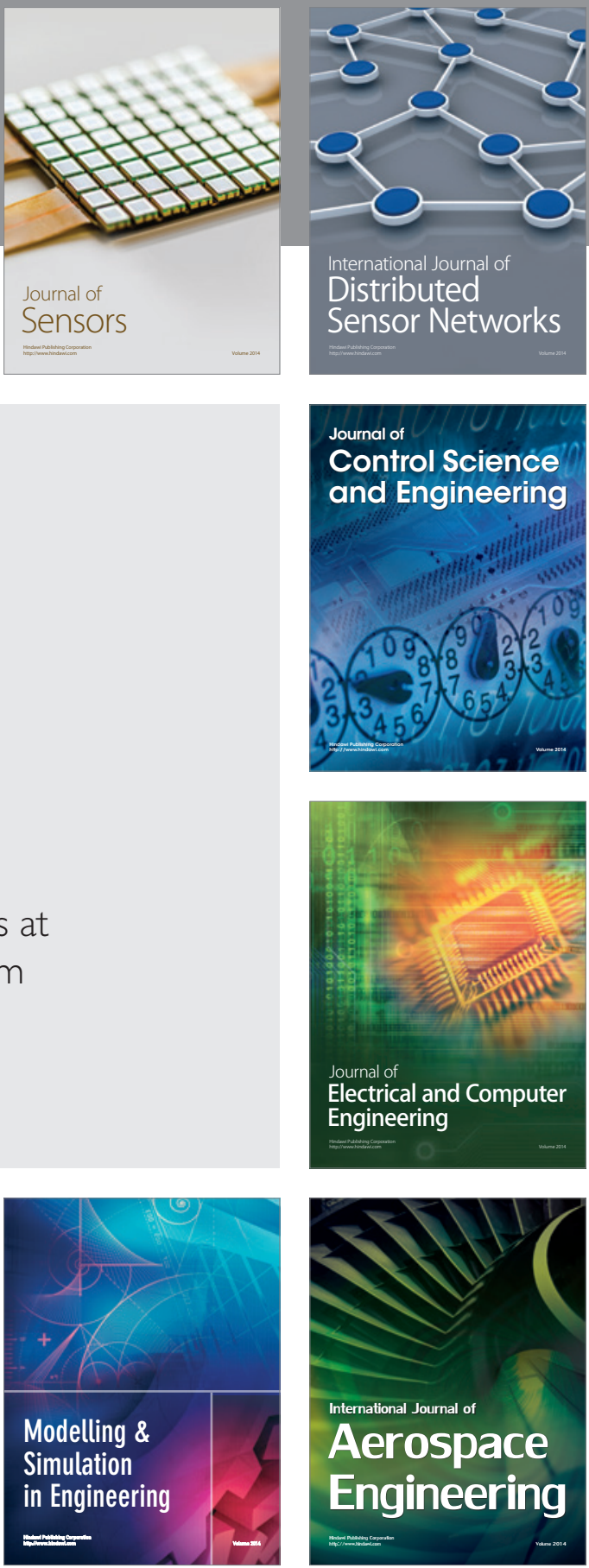

Journal of

Control Science

and Engineering
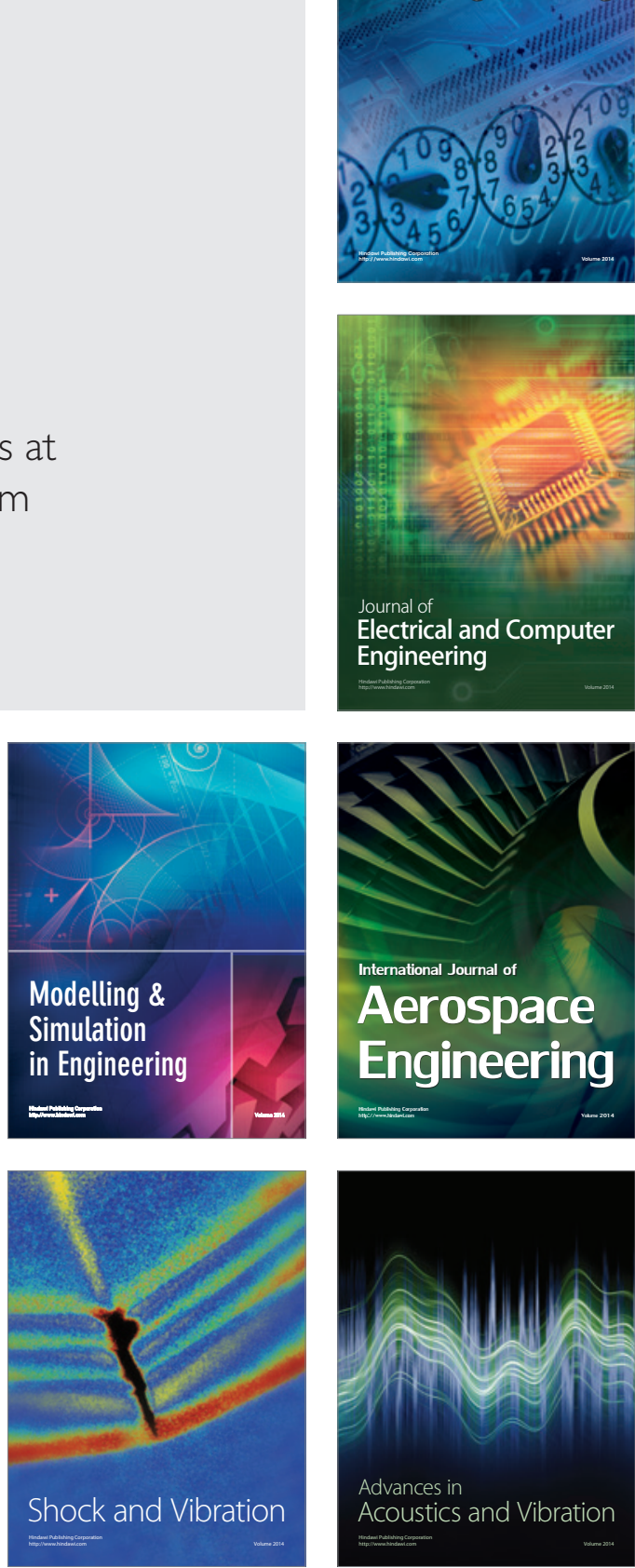